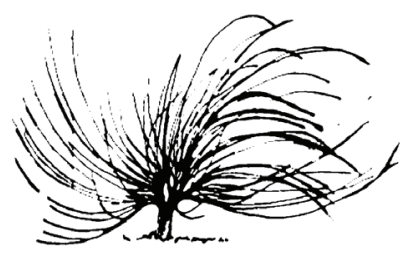

\title{
Redes sociales y comunicación
} universitaria: los desafíos de la Universidad Veracruzana en la época de internet Social Networks and University Communication: The Challenges of the Universidad Veracruzana in the Era of the Internet

\author{
Raciel D. Martínez Gómez ${ }^{l}$
}

\begin{abstract}
Resumen
El uso de internet y las redes sociales ha transformado el mundo de la comunicación. El empoderamiento ciudadano a través de estas tecnologías ha sido muy polémico. Y para las universidades públicas en México no es la excepción, porque el tema cobra especial importancia política y social. Para ello este artículo tiene el propósito de reflexionar las múltiples aristas del epifenómeno. Por un lado, la reconversión del uso debe ubicarse en el contexto de las causas sociológicas que determinan estas actitudes frente a todo lo que se llame autoridad. Una segunda reflexión es de carácter conceptual y está relacionada con la coyuntura económica y política que viven las universidades en México y en específico el estado de Veracruz. Y una tercera reflexión es en términos epistemológicos, ya que prevalece un enfoque tradicionalista de la comunicación, el cual es vital trascender con una visión moderna de lo que está ocurriendo en la etapa de la globalización. El
\end{abstract}

1 Doctor en Sociedades Multiculturales por la Universidad de Granada, España. Director General de Comunicación Universitaria de la Universidad Veracruzana. 
reto, entonces, es superar la satanización de un medio para que internet y las redes sociales puedan incluirse en una política de comunicación universitaria con la justa asertividad para formar una mejor ciudadanía, que dentro de la agenda futura, seguro será uno de los principales desafíos de la educación superior en México, muy probablemente en América Latina y quizás en el mundo.

Palabras clave: redes sociales, internet, comunicación universitaria, empoderamiento y universidades.

\begin{abstract}
The use of the Internet and social media has transformed the world of communication. Citizen empowerment through these technologies has been very controversial, and public universities in Mexico are not an exception because this issue is of special political and social importance. Thus, the article "Social Networks and University Communication: The Challenges of the Universidad Veracruzana in the Era of Internet" is intended to reflect on the many facets of this epiphenomenon. On the one hand, the reconversion of their use must be located in the context of the sociological causes that determine these attitudes towards anything that is considered an authority. A second thought is conceptual in nature and is related to the economic and political situation that universities in Mexico live, specifically in the state of Veracruz. A third reflection is stated in epistemological terms because a traditionalist approach to communication still prevails, which is vital to transcend with a modern vision of what is happening on the stage of globalization. The challenge then is to overcome the demonization of this means for the Internet and social networks to be included in a policy of university communication with fair assertiveness to form better citizens, which, in the future agenda, will surely be one of the main challenges for higher education in Mexico, most likely in Latin America and perhaps in the world.
\end{abstract}

Keywords: social networking, Internet, university communication, empowerment and universities. 


\section{Introducción}

$\mathrm{E}$ propósito del presente artículo es reflexionar en torno a la importancia de incorporar el uso de internet y las redes sociales para la sustancial mejora de la calidad de una política de comunicación universitaria. La serie de reflexiones se hará en el contexto de la Universidad Veracruzana, institución de educación superior pública en México, situación que obliga a describir circunstancias específicas relacionadas a un determinado contexto. Sin embargo, lo aquí expuesto puede compartirse con otras universidades de América Latina que padecen problemáticas similares a raíz de las crisis de los Estado-nación en franco retiro de la rectoría social.

En este trabajo se pretende asumir un enfoque complejo y multicausal, para dejar en claro que no es automático el convencimiento del uso de redes sociales en la política de comunicación universitaria. La propuesta va en el sentido contrario: que la inclusión irreversible de estos novísimos espacios de comunicación vaya acompañada, en consonancia, del concepto mismo de la comunicación. Para ello se esbozaron una serie de planteamientos que permiten construir una estrategia de comunicación pertinente al internet y las redes sociales que, subrayamos, no solo implican la novedad de espacios virtuales con amplio consenso entre la comunidad para favorecer, en todo caso, una interacción que haga posible una comunicación eficiente.

Reconocemos, por un lado, que los planes de comunicación universitaria en una institución de educación superior como la Universidad Veracruzana se desprenden de lineamientos y políticas establecidas y marcadas por la propia universidad a través de una serie de reglamentos que encauzan la labor comunicativa.

Actualmente, de acuerdo con la normatividad universitaria, la Dirección General de Comunicación Universitaria (DGCUV) es la dependencia responsable de planear, diseñar, proponer, dirigir, controlar y evaluar las políticas de imagen institucional, comunicación periodística, estratégica y publicitaria que servirá de base para propagar y transmitir los fines y funciones de la Universidad a través de los distintos medios e instrumentos de comunicación.

Este artículo se desglosa de la siguiente manera. Una primera reflexión será abundar en el debate sobre el empoderamiento de 
la sociedad a través del internet, apuntando hacia posibles causas sociológicas que determinan estas actitudes irreverentes frente a todo lo que se llame autoridad. La segunda reflexión es de carácter conceptual y está relacionada con la coyuntura económica y política que viven las universidades en México y en específico el Estado de Veracruz, y que seguro podrá encontrar resonancias en países de América Latina. Y una tercera reflexión es en términos epistemológicos, porque argumentamos la tesis de que todavía prevalece dentro de los entornos del personal público y universitario un enfoque tradicionalista de la comunicación $\mathrm{y}$, por lo tanto, es vital trascenderlo. Y para concluir exponemos una serie de consideraciones que serían incorporadas para el impulso de la internet en la política de comunicación universitaria.

Cabe destacar que, para esta conversión hacia un uso asertivo del internet y las redes sociales como parte estratégica de la comunicación universitaria, la Dirección General de Comunicación Universitaria (DGCU) y el Centro de Estudios de Opinión y Análisis (CEOA), ambas entidades pertenecientes a la UV, realizaron una encuesta para conocer el consumo de medios de las personas universitarias ${ }^{2}$.

\section{Empoderamiento, inmadurez... y luego}

En esta primera reflexión señalemos posibles causas sociológicas que detonan el empoderamiento de la sociedad a través del internet, y explicar así el inusitado uso que se le da, principalmente, como expresión política.

Parece ya un lugar común que, conforme aparece un medio de comunicación, en determinado periodo, su uso se sataniza de forma moral. Al nuevo medio, como acusan al internet, se le reprocha la liberación y posicionamiento de comportamientos invisibilizados. Este uso de internet y redes sociales en particular ha horizontalizado la relación con el poder (Estulín, 2011; Ubieta 2011), lo que desequilibra los lenguajes, códigos y ritos de comunicación que servían de vínculos entre la sociedad y aquel, en etapas de la historia donde esta relación

2 El método de recolección de datos fue la entrevista. Se entrevistó a personas en las cinco regiones de la UV. El diseño fue a través de un muestreo estratificado en dos etapas, 1. Selección aleatoria de facultades por región, y 2. Selección aleatoria de las personas pertenecientes a la comunidad universitaria de acuerdo con cada tipo de sujeto entrevistado. Levantamiento: 20 y 21 de abril de 2015 . Nivel de confianza del $95 \%$ y error del $+/ 3 \%$. 
era más vertical. Incluso, se ha visto que, con el surgimiento de un nuevo medio de comunicación, se proclama la desaparición o el desplazamiento de otros medios de comunicación en boga.

McLuhan (1964 y 1972), en diferentes momentos, analizó precisamente el miedo al cambio en las diferentes apariciones históricas de los medios de comunicación. Con el internet y las redes sociales no ha ocurrido algo distinto a la moralización de uso que le sucedió a la fotografía, al cine, a la radio y a la televisión. Recordemos tránsitos polémicos dentro de la historia de cada medio como le pasó al cine cuando permuta del mudo al sonoro, y del cine en blanco y negro a cine en color. En este sentido, la aparición y posicionamiento hegemónico del internet y las redes sociales involucra, desde la óptica sociológica, un reacomodo drástico en este mundo desbocado (Giddens, 2000).

Para entender las modificaciones en los procesos de comunicación, en particular vemos lo que pasa con el internet y las redes sociales: su impacto es de tal envergadura que ha rebasado ciertas expectativas y el mismo conocimiento se ha quedado muy lejos de lo que a velocidades extremas se mueven las redes sociales.

Por ello, antes de unirnos de facto a la satanización de las redes sociales, es preferible esforzarnos por comprender la calidad de la comunicación para entender el porqué de pronto, en un lapso muy breve de años, las redes sociales se transformaron en uno de los espacios más acudidos por la ciudadanía. Veamos el caso de México ,en donde es clave referenciar la erosión del pacto político y social de la nación para comprender la efusividad colectiva por las redes. Recordemos que el Estado postrevolucionario funcionó con unas correas de transmisión de consenso a través de lo que se llamó corporativismo y clientelismo (Córdova, 1972). Habrá que decir que dicho consenso gestó el suficiente apoyo en el marco de un Estado sin desarrollo democrático y con una hegemonía basada en el control mediático (Esteinou, 1983). Sin embargo, las crisis económicas de finales del siglo pasado deterioraron el status quo. Y con la globalización esta decadencia de los Estados postnacionalistas, como el mexicano, se acentúa con la aparición de clases medias desgastadas y sin futuros promisorios (Fukuyama, 1989; Giddens, 1999; Huntington, 1998).

¿No será, en todo caso, que la expresión furibunda y exaltada en las redes sociales tenga relación proporcional a la baja calidad de participación y a la baja calidad de comunicación de la ciudadanía 
de la actualidad del país? Al analizar los indicadores de la calidad de la ciudadanía mexicana en específico (INE, 2014), advertimos un descenso alarmante en la confianza en las instituciones y en los medios de comunicación en particular.

Parece, entonces, siguiendo la hipótesis del Instituto Nacional Electoral (INE), que plantea un concepto de ciudadanía nada alentador ${ }^{3}$, el internet y las redes sociales son un espacio de uso derivado de la escasa confianza, o digámoslo, al contrario, de la amplia desconfianza frente a la calidad de comunicación basada en una plataforma mediática subordinada al Estado y a los poderes fácticos. En la región Sur de Veracruz, donde se ubica nuestra universidad, la credibilidad en los medios es del 22\% (INE, 2014), ni siquiera una cuarta parte de la confianza. Esta desconfianza se explica, a su vez, por supuesto, en el contexto histórico de la subordinación de la prensa que arroja una calidad informativa baja, con unos medios acostumbrados a rendir pleitesía al poder ${ }^{4}$.

Nuestra encuesta de Consumo de medios reafirma la poca credibilidad de la ciudadanía y la comunidad universitaria al detectar casi $80 \%$ de desconfianza en los medios.

3 La hipótesis central "es que la ciudadanía en México atraviesa por un complejo proceso de construcción que se puede caracterizar por la desconfianza en el prójimo y en la autoridad, especialmente en las instituciones encargadas de la procuración de justicia; su desvinculación social en redes que vayan más allá de la familia, los vecinos y algunas asociaciones religiosas; y su desencanto por los resultados que ha tenido la democracia” (INE, 2014, p. 19).

4 Recomendamos revisar a Carreño Carlón (2000). 
Redes sociales y comunicación universitaria: los desafíos de la Universidad Veracruzana en la época de internet Social Networks and University Communication: The Challenges of the Universidad Veracruzana in the Era of the Internet

Tabla 1

¿Utiliza los siguientes medios para informarse?

\begin{tabular}{|c|c|c|c|c|c|c|c|c|}
\hline \multicolumn{9}{|c|}{ Medios utilizados para informarse } \\
\hline & $\begin{array}{l}\text { Sí } \\
\%\end{array}$ & $\begin{array}{l}\text { No } \\
\%\end{array}$ & $\begin{array}{c}\text { Todos } \\
\text { los } \\
\text { días } \\
\%\end{array}$ & $\begin{array}{c}\text { Una o dos } \\
\text { veces por } \\
\text { semana } \\
\%\end{array}$ & $\begin{array}{c}\text { Los fines } \\
\text { de } \\
\text { semana } \\
\%\end{array}$ & $\begin{array}{c}\text { Una } \\
\text { vez al } \\
\text { mes } \\
\%\end{array}$ & $\begin{array}{c}\text { Ocasionalmente } \\
\%\end{array}$ & $\begin{array}{c}\text { No } \\
\text { contestó } \\
\%\end{array}$ \\
\hline Televisión & 79.2 & 20.8 & 58.8 & 24.5 & 5.2 & 0.5 & 10.4 & 0.6 \\
\hline La radio & 46.9 & 53.1 & 47.1 & 24.6 & 10.4 & 3.0 & 12.9 & 2.0 \\
\hline $\begin{array}{l}\text { Los periódicos } \\
\text { locales impresos }\end{array}$ & 44.2 & 55.8 & 23.1 & 32.8 & 13.7 & 6.5 & 22.0 & 1.9 \\
\hline $\begin{array}{l}\text { Los periódicos } \\
\text { nacionales } \\
\text { impresos }\end{array}$ & 29.5 & 70.5 & 28.2 & 29.8 & 6.5 & 7.7 & 24.6 & 3.2 \\
\hline Portales de internet & 85.3 & 14.7 & 71.0 & 18.2 & 2.5 & 0.7 & 5.2 & 2.4 \\
\hline $\begin{array}{l}\text { Pláticas con sus } \\
\text { amigos (as), } \\
\text { vecinos (as), } \\
\text { compañeros (as) de } \\
\text { trabajo }\end{array}$ & 80.5 & 19.5 & 71.5 & 15.5 & 2.1 & 0.9 & 7.8 & 2.2 \\
\hline $\begin{array}{l}\text { Redes sociales } \\
\text { (Facebook, Twitter, } \\
\text { etc.) }\end{array}$ & 82.4 & 17.6 & 78.5 & 11.7 & 2.0 & 0.9 & 5.0 & 1.9 \\
\hline Otro & 1.9 & 98.1 & 49.8 & 18.8 & 6.3 & 6.3 & 6.3 & 12.5 \\
\hline
\end{tabular}

Nota: DGCUV y CEOA (2015).

Las respuestas en torno a la utilización de medios entre los sujetos encuestados son muy elocuentes. En la Tabla 1 observamos que el consumo se inclina de manera preferente por los medios electrónicos 
y digitales, y destaca el hábito de establecer su cultura informativa a través de lo digital. La televisión alcanza cerca del $80 \%$, tiene un 79.2 con una frecuencia diaria de $58.8 \%$. La radio curiosamente permanece en un sitio importante entre la comunidad universitaria; se utiliza en un $46.9 \%$ para informarse, y lo interesante es que su frecuencia cotidiana aumenta en un $47.1 \%$. Los periódicos locales impresos tienen un modesto consumo de poco menos de la mitad de la atención universitaria.

Tabla 2

¿En qué lugares tiene acceso a internet?

\begin{tabular}{|c|c|c|}
\hline & Sí \% & No \% \\
\hline Casa & 88.6 & 11.4 \\
\hline Escuela & 59.7 & 40.3 \\
\hline Oficina & 37.0 & 63.0 \\
\hline $\begin{array}{c}\text { Red móvil } \\
\text { (Teléfono celular) }\end{array}$ & 54.2 & 45.8 \\
\hline
\end{tabular}

Nota: DGCUV y CEOA (2015).

Aunque mucho se ha hablado de la diferenciación de acceso a las TIC, entre la comunidad universitaria destaca una cobertura amplia de los servicios de internet. La Tabla 2 arroja que un contundente $96.6 \%$ de estudiantes respondió que sí tiene acceso al internet.

Pero a la pregunta sobre el uso de las redes sociales entre la comunidad universitaria (Tabla 3), los datos corresponden a las tendencias que se dan a nivel mundial. La red a la que más acceso tiene el estudiantado es Facebook con $90.9 \%$ y en segundo término Twitter con $40.1 \%$. Instagram tiene un sorprendente $29.8 \%$ superando a redes con más tiempo como Linkedlin con apenas 9.5\% y MySpace con solo $3.7 \%$ de las preferencias de la comunidad universitaria. Lo que es notable todavía son las diferencias entre los fines de uso. Mientras que para fines sociales o entretenimiento es la mayoría; las redes sociales poco son usadas para el trabajo. Cuando menos lo que pasa con el Facebook que registra $6.1 \%$, es evidente la tendencia de uso que tiene dicha red social. 
Tabla 3

¿Es usted usuario o usuaria de las siguientes redes sociales?

\begin{tabular}{|c|c|c|c|c|c|c|}
\hline \multicolumn{7}{|c|}{ Redes sociales que se usan } \\
\hline & & & \multicolumn{4}{|c|}{ Si la usa, ¿con que fines la usa? } \\
\hline & Sí \% & No $\%$ & $\begin{array}{c}\text { Social o entretenimiento } \\
\text { (amistades, familiares) \% }\end{array}$ & Trabajo \% & Ambos \% & $\begin{array}{c}\text { No } \\
\text { contestó\% }\end{array}$ \\
\hline Facebook & 90.9 & 9.1 & 35.1 & 6.1 & 57.2 & 1.6 \\
\hline Twitter & 40.1 & 59.9 & 54.0 & 11.9 & 33.2 & 0.9 \\
\hline LinkedIn & 9.5 & 90.5 & 15.5 & 59.5 & 25.0 & 0.0 \\
\hline Instagram & 29.8 & 70.2 & 87.2 & 2.5 & 9.1 & 1.2 \\
\hline MySpace & 3.7 & 96.3 & 51.4 & 8.6 & 37.1 & 2.9 \\
\hline Otra & 10.1 & 89.9 & 35.4 & 14.6 & 39.0 & 11.0 \\
\hline
\end{tabular}

Nota: DGCUV y CEOA (2015).

Con este panorama, que es una correlación de fuerzas -proporciones frente a ausencias-, sabemos qué significa el nivel de empoderamiento de una comunidad, sobre todo juvenil, en sociedades en transición política, como las nuestras, en las cuales el concepto mismo de ciudadanía está en progreso y las redes sociales se convierten en espacios de catarsis donde vuelcan el desencanto por las democracias incipientes.

Sabemos, y hasta compartimos en cierto punto, la opinión que cierne sobre el uso inmaduro del internet y las redes sociales en relación con la impotencia ciudadana que desfoga lo que históricamente ha sido una relación autoritaria -verticalismo sin amplias libertades-, en detrimento de la confianza en la autoridad e imagen de las instituciones a nivel general. Empero, esta podría ser solo eso: una idea todavía muy etérea del comportamiento de la comunidad de jóvenes de sociedades en plena efervescencia democrática, y no por lo anterior descartar el uso dentro de las estrategias de comunicación universitaria; al contrario, más bien desde la universidad construir una cultura más responsable en el uso del internet. Valdría la pena también ubicar este empoderamiento de masas -o colectivo-, en redes sociales en una complicada coyuntura política y económica, la de México y Veracruz, en concreto, lo que ofrece luces para cualquier tipo de política de comunicación universitaria. 


\section{Convulsa coyuntura política y económica}

La situación que viven las universidades públicas en México es sui generis. Por un lado la Organización para la Cooperación y el Desarrollo Económicos (OCDE) afirma que México es el país que más gasta en educación. Sin embargo, por otro, las instituciones de educación superior han presionado al respecto, contradiciendo la afirmación de la OCDE. Por ejemplo, la Universidad Nacional Autónoma de México (UNAM) y la Asociación Nacional de Universidades e Instituciones de Educación Superior (ANUIES) demandaron un aumento urgente al presupuesto de educación superior, denunciaron el decrecimiento del subsidio, los rectores mismos hicieron declaraciones pidiendo un incremento y, ANUIES exige que se incremente en al menos a un diez por ciento el presupuesto a nivel federal (Gómez, 2013; Luna, 2015).

A la par que sufren el retiro de los apoyos del Estado, que debiesen recibir por ley, se agrega un ambiente de poderes fácticos que empeora la condición paupérrima de las instituciones dedicadas a la educación superior. Se trata de una circunstancia totalmente asimétrica en donde parece haber una pugna de fuerzas fácticas que intentan desacreditar el proyecto de educación superior pública a como dé lugar.

En este sentido, independientemente de los grupos que riñen y los que apoyan la reforma educativa propuesta por el actual gobierno federal, se generó un ambiente mediático adverso a este tipo de proyectos. Este ambiente mediático es, por un lado, resultante de la revolución tecnológica generada por la globalización que ha impulsado el nacimiento de proyectos de comunicación sin representar un volumen económico oneroso para constituirse en empresas.

Al mismo tiempo, y esa es una característica de nuestro país, el ambiente mediático que se desprende de los últimos quince años es consecuencia de la corrupción y opacidad de los aparatos estatales en plena crisis: hay un crack económico, perono inversión públicani privada; se tiene una amplia deuda con pago de intereses exorbitantes, un piso de jubilados insostenible, una burocracia inoperante y rotas las correas de consenso tradicionales como el clientelismo y el corporativismo, lo que provoca una incertidumbre política sin precedentes.

El modelo subordinado de prensa en México sostuvo un esquema económico proteccionista, "vigente desde los años veinte hasta la 
primera mitad de los ochentas, que al ser aplicado a las empresas mediáticas con las características de discrecionalidad propias de los vacíos legales descritos, generó relaciones de corrupción, dependencia y subordinación del Estado con empresarios y profesionales de la información" (Carreño, 2000). En esta corrupción se abre un abanico amplio de formas de canalizar recursos públicos como apoyos financieros estatales o créditos preferenciales a la prensa o medios tradicionales.

El Estado mexicano ha prohijado una diversidad de relaciones que le permiten mantener cooptados a periodistas con prebendas: estímulos fiscales para regularizar deudas acumuladas; dotación subsidiada de insumos; publicidad estatal asignada discrecionalmente sin criterios transparentes donde se indique el valor social del medio; condonación de deudas acumuladas por servicios de salud; asignación de emolumentos pecuniarios a manera de salarios o complemento de salarios; concesiones discrecionales a empresas formadas por periodistas de igualas periódicas; y la ominosa creación de "fondos de reptiles" que se canalizan en las eufemísticas empresas representantes (Carreño, 2000).

Insistimos que, sobre todo en los últimos quince años, se generó una burbuja mediática, la cual amplió esa especie de prensa subsidiada sin control ninguno. Si anteriormente se detectaba una prensa seriamente limitada en sus libertades, resultó que este boom, en donde el poder gozó de impunidad para deslizar canonjías de forma opaca y discrecional; ahora esta prensa de tipo oficialista creció en volumen, a grados de tratar de justificar actividades comunicativas sin resonancia social -pocos medios de comunicación publican de forma certificada sus tirajes o su rating-.

El problema mayor es que, al quedar en crisis estos aparatos estatales, se desinfla la burbuja mediática, insostenible para el sistema, y este boom mediático se transforma en una serie de medios de comunicación que ya no reciben dicho subsidio. Esto trae consigo un estado de vulnerabilidad para el resto de las instituciones, como las dedicadas a la educación superior, buscadas y prácticamente extorsionadas por algunos medios de comunicación que no tienen formas de sobrevivir comercialmente, porque ya se hallan fuera del circuito de subsidios.

Este contexto asimétrico provocó problemas en las instituciones de educación superior, porque enfrentan una serie de crisis al mismo 
tiempo. Por un lado, ya decíamos, el retiro paulatino de los apoyos del gobierno federal, y por otro, un ambiente mediático que persigue la noticia negativa y que golpea a las instituciones estigmatizando, sobre todo, el comportamiento estudiantil ligándolo a la criminalidad y al desorden público.

A esta convulsa coyuntura se le agrega un terminante cambio en el consumo de medios de información entre la comunidad universitaria -es decir, la transformación radical en los procesos de información-, en donde las cifras revelan un elocuente hábito por los llamados medios electrónicos y digitales, situación que modifica la política de comunicación de cualquier institución de educación superior.

El consumo de medios descarta a los medios de información impresos y destaca como medio privilegiado a la televisión y, de manera preponderante, aparece en este habitus informativo el uso de las redes sociales. De ahí, insistimos, las instituciones de educación superior tendrán que replantear su mirada, enfoque y acciones a un habitus informativo que ya transversalizó a las comunidades universitarias.

Esto, aunque de compleja factura, es fácil de admitir si no fuera porque en el telón de fondo existe todavía una serie de prejuicios relacionados íntimamente con un punto de partida epistemológico tradicional que limita la comprensión del estado actual de los medios de comunicación.

\section{Nuevas dinámicas perceptoras}

Ahora bien, por otra parte, esto no significa que la institución tenga como camisa de fuerza una política de comunicación establecida. No puede ser, porque entonces se convierte, en los escenarios de los vaivenes políticos y en las aceleradas y tremendas transformaciones tecnológicas, en una política reactiva ante lo que ocurre en el ambiente mediático, con escaso margen de mantener una propuesta institucional -y entonces limitarse a la fatalidad que se ha convertido en una tendencia: manuales y estrategias para una comunicación en crisis-. Y no, las instituciones de educación superior también deben transformar su mirada y postura ante las novedades de la comunicación que suponen, entre otras cosas, el uso del internet y las redes sociales.

En el fondo, lo que tratamos de decir es que, si bien aceptamos ese sentido de plegarse a la política de una institución, se tiene que 
ser flexible para derivar y admitir que vivimos en un mundo diferente, donde los esquemas de comunicación han cambiado dramáticamente.

Manifestémoslo de esta manera: las instituciones de educación superior tendrán que superar esos esquemas de mitad del Siglo XX, en donde se admitía el poder narcotizante de la comunicación. En otros espacios hemos sostenido cómo la comunicación ha registrado una serie de desplazamientos que conviene incorporar al corpus institucional (Martínez, 2014 y 2015).

Todavía en los entornos burocráticos de las administraciones actuales, tanto en el nivel estatal como universitario, se detecta una tradicional demonización de la cultura de masas y un poco entendimiento a lo que se llama sociedad red (Castells, 2000). Hay una visión simplificada que no advierte la revolución en los esquemas comunicativos.

Cuando se habla de las redes sociales, lo que prevalece son criterios moralizantes en torno al mal uso que se les da y una recriminación generacional que ya en anteriores cambios tecnológicos se dio. Hay una reprimenda a las culturas juveniles en torno a su debilidad axiológica para usar las nuevas herramientas de comunicación y se duda de su densidad ciudadana. Esta mirada tradicional está rebasada para explicar la extensa diferenciación social y cultural generada por la globalización.

Dentro de estas nuevas dinámicas perceptoras volvemos a la encuesta de Consumo de medios para resaltar la tendencia a recurrir a fuentes informativas diferentes a las establecidas en una plataforma de comunicación del siglo pasado. Cuando se les precisa en la pregunta, si utiliza a las redes sociales para informarse (Tabla 4), por regiones, resulta que los porcentajes son muy altos frente al bajísimo porcentaje que tienen los periódicos impresos. De las cinco, tres regiones están alrededor del noventa por ciento: Veracruz, 90.1\%, Córdoba-Orizaba, $89.1 \%$ y Coatzacoalcos $88.5 \%$. 


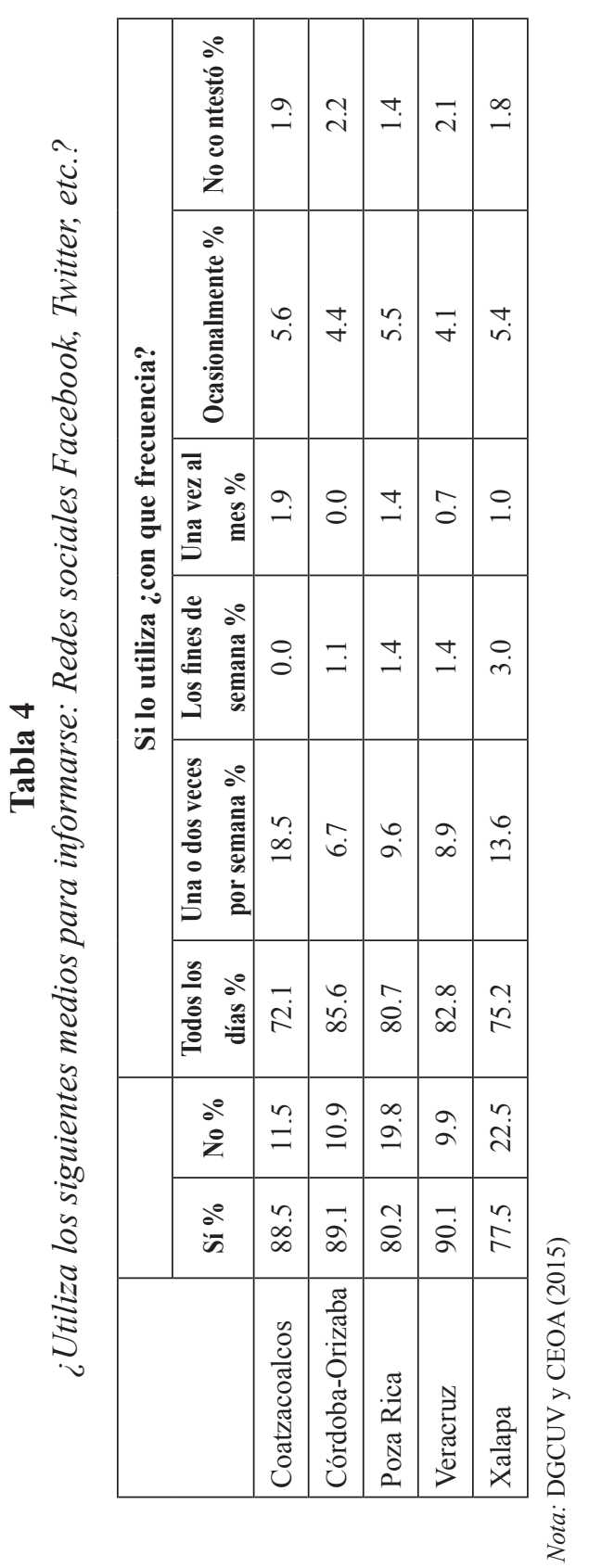


Este vuelco de la comunidad universitaria para el uso del internet sería consecuencia directa del magma de desconfianza ciudadana en los medios de comunicación tradicionales, que nos obliga a asumir un enfoque de mirada más compleja, que nos permita una lectura menos reactiva y, en todo caso, insistir en la conversión de los espacios que ofrecen internet y las redes sociales para construir el presente y futuro próximos de la comunicación universitaria.

\section{Una nueva cultura de comunicación}

Como una consideración final-no cerrada por supuesto-, podemos afirmar que el uso del internet y las redes sociales mejorará la calidad de una política de comunicación universitaria. Las circunstancias específicas relacionadas con las crisis de las universidades en América Latina exigen, precisamente, modificar los canales informativos, y el del internet y las redes sociales garantizan cierto impacto en nichos de la comunidad universitaria.

Hay múltiples causas sociológicas que determinan el empoderamiento de la sociedad a través del internet, entre ellas, factores políticos que justifican el tono. Apostamos, entonces, porque la problemática del internet es su uso, más no el medio en sí. Corresponde a las instituciones, como las universidades, revertir dicha utilización en favor o en construcción de una cultura del debate, del establecimiento de la diferencia con responsabilidad, que conlleve un respeto, tolerancia, ética y argumentación sustentada.

No satanicemos y, en cambio, aprovechemos las características de estos nuevos lenguajes comunicativos. Confiamos en que pronto habrá un equilibrio luego de un desfogue que alcanzaría la madurez ciudadana. Es una época convulsa; pero, por ello mismo, demanda el desafío creativo de las instituciones.

En este sentido, la DGCU establece como objetivos generales un posicionamiento de calidad permanente de la imagen institucional que no es más que la visibilización de las actividades universitarias de investigación, difusión artística, vinculación y gestión en la opinión pública. La imagen institucional debe ser reflejo de una vida académica tan diversa en su interior, mostrando lo que se gesta en las facultades, institutos y programas de orden científico, técnico, social y artístico; $\mathrm{y}$, asimismo, tan rica en su exterior, con los grupos egresados que han 
desarrollado su talento, ejemplos de éxito que posiciona a la UV y coadyuva a la apertura de campos laborales. Que dicha construcción de imagen de confianza se dé a nivel regional, en la medida que la UV intensifique la visibilización de su presencia en las regiones, de esa manera no solo se promocionará su oferta académica, sino se establecerán puentes entre los diversos actores sociales locales, para encontrar, en colaboraciónn las vocaciones regionales más urgentes. Y se intensificarán circuitos de comunicación para la comunidad estudiantil y se promoverá la sinergia del capital comunicacional universitario.

De ahí que la comunicación universitaria se sitúe en los programas estratégicos (Ladrón de Guevara, 2013) de la UV para lograr una presencia en el entorno con pertinencia e impacto social, la modernización de los diferentes medios de comunicación universitarios (prensa, radio y tv) y que la comunicación coadyuve a una gestión universitaria más responsable y con transparencia.

El internet y las redes sociales pueden incluirse, para ello, en una política de comunicación universitaria con la justa asertividad para formar mejores ciudadanos y ciudadanas: ese es el desafío por venir.

\section{Referencias}

Carreño, J. (Febrero, 2000). Cien años de subordinación. Un modelo histórico de la relación entre prensa y poder en México en el siglo XX. Sala de Prensa, 2(16). Recuperado de http://www.saladeprensa.org/art102.htm

Castells, M. (2000). La era de la información. Economía, sociedad y cultura (Vol. 2, El poder de la identidad). Madrid: Alianza.

Córdova, A. (1972). La formación del poder político en México. México: Era.

Esteinou, J. (1983). Los medios de comunicación y la construcción de la hegemonía. México: Nueva Imagen.

Estulin, D. (2011). Desmontando a WikiLeaks. Barcelona: Planeta.

Fukuyama, F. (1989). El fin de la historia y el último hombre. México: Planeta.

Giddens, A. (1999). Un mundo desbocado. Los efectos de la globalización en nuestras vidas. Madrid: Taurus.

Gómez, N. (viernes 8 de 2013). Recursos para educación no son 'dádivas': ANUIES. El Universal. México. Recuperado de http:// 
www.eluniversal.com.mx/nacion-mexico/2013/recursos-para-educacion-no-son-39dadivas-39-anuies-964156.html

Huntington, S. P. (1998). El choque de las civilizaciones y la reconfiguración del orden mundial. México: Paidós.

Instituto Nacional Electoral. (2014). Informe del país sobre la calidad de la ciudadanía en México. México: INE-Colegio de México.

Ladrón de Guevara, S. (2013). Programa de Trabajo. Tradición e innovación. México: Universidad Veracruzana. Recuperado de http:// www.uv.mx/juntagob/designacion-2013-2017/

Luna, E. (28 de enero, 2015). Pide UNAM-ANUIES aumento urgente de presupuesto en educación superior. El Punto Crítico. México. Recuperado de http://www.elpuntocritico.com/opinion/ columnistas/130-columna-asi-le-fue-al-presidente/107489-pide-unam-anuies-aumento-urgente-de-presupuesto-en-educaci\%C3\%B3n-superior.html

Martínez, R. D. (2014). Comunicación y TIC: De la masa a la red, un cambio en el paradigma. En A. Ramírez-Martinell y M. A. Casillas (Eds.), Háblame de TIC: Tecnología Digital en la Educación Superior (85-106). Argentina: Brujas.

Martínez, R. D. (Marzo- mayo, 2015). Medios y opinión pública. Las veedurías de medios en época de crisis. Razón y Palabra, 89. Recuperado de http://www.razonypalabra.org.mx/N/N89/ RE89/01_Martinez_R89.pdf

McLuhan, M. (1964). Understanding Media: The Extensions of Man. Nueva York: McGraw-Hill.

McLuhan, M. (1972). La galaxia Gutemberg. Génesis del homo typographycus. Madrid: Aguilar.

Ubieta, E. (2011). WikiLeaks: Sobre la libertad de información y la descontextualización de la historia. Recuperado de http://a-isla-desconocida.blogspot.com/2011/01/wikileaks-sobre-la-libertad-de.html 